\title{
Local Exponential Regulation of Nonholonomic Systems in Approximate Chained Form with Applications to Off-Axle Tractor-Trailers
}

\author{
Bao-Li Ma \\ The Seventh Research Division, BeiHang University, Beijing 100191, China \\ Correspondence should be addressed to Bao-Li Ma, mabaoli@buaa.edu.cn
}

Received 21 January 2011; Revised 29 April 2011; Accepted 2 June 2011

Academic Editor: G. Muscato

Copyright (c) 2011 Bao-Li Ma. This is an open access article distributed under the Creative Commons Attribution License, which permits unrestricted use, distribution, and reproduction in any medium, provided the original work is properly cited.

\begin{abstract}
Most of drift-less nonholonomic systems cannot be exactly converted to an nonholonomic chained form, a wealth of design tools developed for the control of nonholonomic chained form are thus not directly applicable to such systems. Nevertheless, there exists a class of systems that may be locally approximated by the nonholonomic chained form around certain equilibrium points. In this work, we propose a discontinuous and a smooth time-varying control laws respectively for the approximated nonholonomic chained form, guaranteeing local exponential convergence of state to the desired equilibrium point. An tractor towing off-axle trailers is taken as an example to illustrate the approaches.
\end{abstract}

\section{Introduction}

The so-called nonholonomic chained form (NCF) has motivated many research activities for about twenty years [1]. Several features such as flatness $[2,3]$, homogeneity, and nilpotency make the NCF especially attractive to work with. These properties have been used for designing control laws to achieve several control objects such as point stabilization and trajectory tracking. Concerning the point stabilization problem of NCF, which is difficult due to Brokett's wellknown obstruction [4], a number of approaches have been developed, which may be roughly classified into discontinuous time-invariant feedback [5-7], continuous time-varying feedback [8-10], and hybrid feedback [11, 12]. The stabilization problems of NCF with parameter uncertainties and perturbation terms have also been attacked in recent years [1317]; however, most of these researches require that the perturbation terms satisfy certain cascaded conditions, which may be very restrictive and thus rule out many interesting examples such as the tractor-trailers with off-axle hitching [18] and the ball-plate systems [19]. It is also mentioned that the dynamics of many nonholonomic driftless systems can be approximated by NCF locally around certain equilibrium points. In [18], a time-varying continuous stabilizing scheme was proposed for such approximate NCF, achieving local exponential stability of the closed-loop system around the selected equilibrium point.

In this paper, we consider the local exponential regulation problem of a class of nonholonomic systems convertible to the approximate NCF. By employing a discontinuous and/or a smooth time-varying coordinate transformations, the approximate NCF is converted to linear perturbed ones with the perturbation terms being second or higher orders of the converted states; then a discontinuous time-invariant and/ or a smooth time-varying control laws are derived respectively, guaranteeing that the state of the approximate NCF converges to zero exponentially, provided the norm of an initial state is sufficiently small. Compared with the control law presented in [18] which is continuous but not differentiable, the time-varying control law proposed in this paper is smooth and can be easily extended to deal with input dynamics.

The paper is organized as follows. Section 2 defines a class of systems that can be approximated by NCF. In Section 3, a discontinuous time-invariant and a smooth time-varying controllers are developed to stabilize the approximate NCF. In Section 4, a tractor-trailer with off-axle hitching is taken as an example to illustrate the effectiveness of the proposed controllers. Section 5 concludes the paper. 


\section{A Class of Approximated Chained Forms}

Consider the following nonlinear system represented by

$$
\begin{gathered}
\dot{x}_{0}=u_{0}, \\
\dot{x}=g_{0}(x) u_{0}+g_{1}(x) u_{1},
\end{gathered}
$$

where $x_{0} \in \mathfrak{R}, \quad x \in \mathfrak{R}^{n}$ are state variables and $u_{0} \in \mathfrak{R}, u_{1} \in$ $\mathfrak{R}$ are control inputs. The control vector fields $g_{0}(x) \in$ $\mathfrak{R}^{n}, \quad g_{1}(x) \in \mathfrak{R}^{n}$ are supposed to have the following forms:

$$
g_{0}(x)=A x+R_{2}(x), \quad g_{1}(x)=b+R_{1}(x),
$$

where

$$
A=\left[\begin{array}{ccccc}
0 & 0 & \ldots & 0 & 0 \\
1 & 0 & \ldots & 0 & 0 \\
0 & 1 & \ldots & 0 & 0 \\
\vdots & \vdots & \ddots & \vdots & \vdots \\
0 & 0 & \ldots & 1 & 0
\end{array}\right], \quad b=\left[\begin{array}{c}
1 \\
0 \\
0 \\
\vdots \\
0
\end{array}\right]
$$

$R_{1}(x) \in \mathfrak{R}^{n}$ denotes the first-or higher-order residual term of $x$ and $R_{2}(x) \in \mathfrak{R}^{n}$ the second or higher-order residual term of $x$ in the state domain $D$; or, say more precisely, there exist three positive constants $r, r_{1}$, and $r_{2}$ such that $R_{1}(x), R_{2}(x)$ are bounded by

$$
\left\|R_{1}(x)\right\|_{2} \leq r_{1}\|x\|_{2}, \quad\left\|R_{2}(x)\right\|_{2} \leq r_{2}\|x\|_{2}^{2}
$$

in the compact set $\Omega=\left\{x:\|x\|_{2} \leq r\right\} \in D$.

System (1)-(2) is called the approximate NCF if (3)-(5) are satisfied.

Remark 1. Without loss of generality, it is specially assumed in (4) that $\{A, b\}$ is in the canonical controllable form. For the controllable pair $\{A, b\}$ not in this form, one can always find a linear state transformation to convert it to this form.

Remark 2. It is noted that the approximate NCF (1)-(2) is not flat with certain defects [2] and thus difficult to control.

The approximate NCF represents a large class of nonholonomic systems that cannot be converted to NCF in which $R(x)=0$. The examples of approximate NCF include tractor-trailers with off-axle hitching [18] and the ball-plate systems [19].

\section{Local Exponential Regulation of the Approximate NCF}

In this section, a discontinuous and a smooth time-varying control laws are derived to solve the local exponential regulation problem of the approximate NCF defined in (1)-(5).

3.1. Local Exponential Regulation of the Approximate NCF for $x_{0}(0) \neq 0$. The control law for the first control input is designed as

$$
u_{0}=-k_{0} x_{0}
$$

with $k_{0}>0$, so that $x_{0}(t)=x_{0}(0) e^{-k_{0} t} \neq 0\left(\forall x_{0}(0) \neq 0,0 \leq\right.$ $t<\infty)$.

Substituting (6) into (2) results

$$
\dot{x}=-k_{0} x_{0}\left(A x+R_{2}(x)\right)+\left(b+R_{1}(x)\right) u_{1} .
$$

Inspired by the well-known $\sigma$-process [5], we introduce the following discontinuous state transformation:

$$
y=T^{-1}\left(x_{0}\right) x, \quad x=T\left(x_{0}\right) y
$$

with

$$
\begin{aligned}
T\left(x_{0}\right) & =x_{0}^{m} \operatorname{diag}\left\{1, x_{0}, x_{0}^{2}, \ldots, x_{0}^{n-1}\right\}, \\
T^{-1}\left(x_{0}\right) & =x_{0}^{-m} \operatorname{diag}\left\{1, x_{0}^{-1}, x_{0}^{-2}, \ldots, x_{0}^{-(n-1)}\right\},
\end{aligned}
$$

and $m$ a positive integer to be determined.

Remark 3. The discontinuous coordinate transformation (8)-(9) is a generalization of the ordinary $\sigma$-process proposed in [5] with $m=0$ for NCF. It is seen in what follows that the term $x_{0}^{m}$ with $m>0$ is crucial for the controller design of the approximate NCF.

The transformation matrix $T\left(x_{0}\right)$ is clearly nonsingular for $x_{0}(0) \neq 0,0 \leq t<\infty$.

The dynamics of the transformed state $y$ can be derived as

$$
\begin{aligned}
\dot{y}= & T^{-1}\left(x_{0}\right) \dot{x}+\frac{d}{d t}\left(T^{-1}\left(x_{0}\right)\right) x \\
= & -k_{0} x_{0} T^{-1}\left(x_{0}\right) A T\left(x_{0}\right) y+T^{-1}\left(x_{0}\right) b u_{1} \\
& +T^{-1}\left(x_{0}\right)\left(-k_{0} x_{0} R_{2}+R_{1} u_{1}\right)+\frac{d}{d t}\left(T^{-1}\left(x_{0}\right)\right) T\left(x_{0}\right) y .
\end{aligned}
$$

Direct calculation reveals that

$$
\begin{aligned}
& T^{-1}\left(x_{0}\right) b=x_{0}^{-m} b, \\
& x_{0} T^{-1}\left(x_{0}\right) A T\left(x_{0}\right)=A, \\
& \frac{d}{d t}\left(T^{-1}\left(x_{0}\right)\right) T\left(x_{0}\right) \\
& \quad=k_{0} \operatorname{diag}\{m, m+1, m+2, \ldots, m+n-1\} .
\end{aligned}
$$

Substituting the above identities into (10) results in

$$
\dot{y}=A_{1} y+x_{0}^{-m} b u_{1}+T^{-1}\left(x_{0}\right)\left(-k_{0} x_{0} R_{2}+R_{1} u_{1}\right),
$$

where

$$
A_{1}=k_{0}(-A+\operatorname{diag}\{m, m+1, m+2, \ldots, m+n-1\}) .
$$


Remark 4. As $\{A, b\}$ is controllable, so is $\left\{A_{1}, b\right\}$; hence, the eigenvalues of $A_{1}-b K$ can be arbitrarily assigned by selecting the control gain $K$.

The second control input is designed as

$$
u_{1}=-x_{0}^{m} K y=-x_{0}^{m} K T^{-1}\left(x_{0}\right) x,
$$

where $K=\left[k_{1}, k_{2}, \ldots, k_{n}\right]$ is a control gain row vector such that $A_{1}-b K$ is Hurwitz.

The closed-loop system of (12) and (14) becomes

$$
\begin{aligned}
\dot{y} & =\left(A_{1}-b K\right) y-T^{-1}\left(x_{0}\right)\left(k_{0} x_{0} R_{2}+R_{1} x_{0}^{m} K y\right) \\
& =\left(A_{1}-b K\right) y+R,
\end{aligned}
$$

where

$$
R=-T^{-1}\left(x_{0}\right)\left(k_{0} x_{0} R_{2}+K y x_{0}^{m} R_{1}\right) .
$$

System (15) is a linear stable one perturbed by a residual term $R$. If $R$ can be shown to be second or higher order of $\|y\|$, then (15) is locally exponential stable. by

In view of (5), the converted residual term $R$ is bounded

$$
\begin{aligned}
\|R\|_{2} \leq & \left\|T^{-1}\right\|_{2}\left\|k_{0} x_{0} R_{2}+K x_{0}^{m} y R_{1}\right\|_{2} \\
\leq & \left\|T^{-1}\right\|_{2}\left(r_{2} k_{0}\left|x_{0}\right|\|x\|_{2}^{2}+r_{1}\|K\|_{2}\left|x_{0}\right|^{m}\|x\|_{2}\|y\|_{2}\right) \\
\leq & \left(r_{2} k_{0}\left|x_{0}\right|\left\|T^{-1}\right\|_{2}\|T\|_{2}^{2}\right. \\
& \left.+r_{1}\|K\|_{2}\left|x_{0}\right|^{m}\left\|T^{-1}\right\|_{2}\|T\|_{2}\right)\|y\|_{2}^{2} \\
= & h\left(\left|x_{0}\right|\right)\|y\|_{2}^{2}
\end{aligned}
$$

with $h\left(\left|x_{0}\right|\right)$ defined as

$$
\begin{aligned}
h\left(\left|x_{0}\right|\right) \cong & r_{2} k_{0} \max \left\{\left|x_{0}\right|^{m+1},\left|x_{0}\right|^{m-n+2}\right\} \max \left\{1,\left|x_{0}\right|^{2(n-1)}\right\} \\
& +r_{1}\|K\|_{2} \max \left\{\left|x_{0}\right|^{m},\left|x_{0}\right|^{m-(n-1)}\right\} \\
& \times \max \left\{1,\left|x_{0}\right|^{n-1}\right\} .
\end{aligned}
$$

As $\left|x_{0}\right| \leq\left|x_{0}(0)\right|, h\left(\left|x_{0}\right|\right)$ is thus bounded uniformly with $t$ provided $m-(n-1) \geq 0$. In view of the facts that $A_{1}-b K$ is Hurwitz and $\lim _{\|y\|_{2} \rightarrow 0}\left(\|R\|_{2} /\|y\|_{2}\right)=0$, system (15) is thus locally exponential stable by Lyapunov indirect approach [20].

The above analysis is summarized as the following proposition.
Proposition 1. Suppose that $0<\left|x_{0}(0)\right|<\infty, k_{0}>0, m \geq n-$ $1, K$ is selected such that $A_{1}-b K$ is Hurwitz then the following control law

$$
u_{0}=-k_{0} x_{0}, \quad u_{1}=-x_{0}^{m} K y=-x_{0}^{m} K T^{-1}\left(x_{0}\right) x
$$

guarantees that the states $x_{0}(t), u_{0}(t)$ globally converge to zero and $x(t), u_{1}(t)$ converge to zero exponentially for a sufficiently small $\|y(0)\|_{2}$.

Proof. It is obvious that $x_{0}(t)=x_{0}(0) e^{-k_{0} t}, u_{0}(t)=-k_{0} x_{0}(t)$ globally converge to zero exponentially. As $A_{1}-b K$ is Hurwitz and $\|R\|_{2} \leq h\left(\left|x_{0}\right|\right)\|y\|_{2}^{2}$ with $h\left(\left|x_{0}\right|\right)$ uniformly bounded with $t$, the closed-loop system (15) is locally exponential stable, implying that $y(t), x(t)=T\left(x_{0}(t)\right) y(t)$ and $u_{1}(t)=-x_{0}^{m}(t) K y(t)$ are all convergent to zero exponentially for a sufficiently small $\|y(0)\|_{2}$.

Proposition 1 is only applicable for $x_{0}(0) \neq 0$. In the case of $x_{0}(0)=0$, the proposed control law fails to work as the transformation matrix $T\left(x_{0}\right)$ becomes singular. This problem may be solved by introducing a switching mechanism that first drives $x_{0}$ away from zero in finite time and then switches to the control law (19) to achieve local exponential regulation for an arbitrarily $x_{0}(0)$ and a sufficiently small $\|x(0)\|_{2}$. However, such switching control law is discontinuous and may cause problems when the velocity input dynamics is included in the model since the discontinuities of velocity inputs lead to infinite accelerations.

In the next subsection, the controller (19) is modified to be smooth and time varying for an arbitrary $x_{0}(0)$ so that the acceleration signals are bounded.

3.2. Local Exponential Regulation of the Approximate NCF for an Arbitrary $x_{0}(0)$. The control law for the first control input is designed as

$$
u_{0}=-k_{0} \alpha(t)-\bar{k}_{0}\left(x_{0}-\alpha(t)\right)
$$

with $\alpha(t)=\alpha_{0} e^{-k_{0} t}, \alpha_{0} \neq 0, \bar{k}_{0}>k_{0}>0$.

Let $e_{0}(t)=x_{0}(t)-\alpha(t)$; then $\dot{e}_{0}(t)=u_{0}(t)-\dot{\alpha}(t)=$ $-\bar{k}_{0} e_{0}(t)$, so that $e_{0}(t)=e_{0}(0) e^{-\bar{k}_{0} t}, x_{0}(t)=\alpha(t)+e_{0}(t)=$ $\alpha(t)+e_{0}(0) e^{-\bar{k}_{0} t}, u_{0}(t)=-k_{0} \alpha(t)-\bar{k}_{0} e_{0}(0) e^{-\bar{k}_{0} t}$, and $e_{0}(t) / \alpha(t)=\left(e_{0}(0) / \alpha_{0}\right) e^{-\left(\bar{k}_{0}-k_{0}\right) t}$ are all globally convergent to zero exponentially.

Now we introduce the following smooth time-varying state transformation:

$$
y=T^{-1}(\alpha) x, \quad x=T(\alpha) y
$$

with

$$
\begin{aligned}
T(\alpha) & =\alpha^{m} \operatorname{diag}\left\{1, \alpha, \alpha^{2}, \ldots, \alpha^{n-1}\right\}, \\
T^{-1}(\alpha) & =\alpha^{-m} \operatorname{diag}\left\{1, \alpha^{-1}, \alpha^{-2}, \ldots, \alpha^{-(n-1)}\right\},
\end{aligned}
$$

and $m$ a positive integer to be determined.

As $\alpha_{0} \neq 0$, the transformation matrix $T(\alpha)$ is clearly nonsingular for all $0 \leq t<\infty$. 
The dynamics of the transformed state $y$ can be derived as

$$
\begin{aligned}
\dot{y}= & T^{-1}(\alpha) \dot{x}+\frac{d}{d t}\left(T^{-1}(\alpha)\right) x \\
= & u_{0} T^{-1}(\alpha) A T(\alpha) y+T^{-1}(\alpha) b u_{1} \\
& +T^{-1}(\alpha)\left(u_{0} R_{2}+R_{1} u_{1}\right)+\frac{d}{d t}\left(T^{-1}(\alpha)\right) T(\alpha) y \\
= & -k_{0} \alpha\left(1+\frac{\bar{k}_{0} e_{0}}{k_{0} \alpha}\right) T^{-1}(\alpha) A T(\alpha) y+T^{-1}(\alpha) b u_{1} \\
& +T^{-1}(\alpha)\left(-k_{0} \alpha\left(1+\frac{\bar{k}_{0} e_{0}}{k_{0} \alpha}\right) R_{2}+R_{1} u_{1}\right) \\
& +\frac{d}{d t}\left(T^{-1}(\alpha)\right) T(\alpha) y .
\end{aligned}
$$

Simple calculation reveals that

$$
\begin{aligned}
& T^{-1}(\alpha) b=\alpha^{-m} b \\
& \alpha T^{-1}(\alpha) A T(\alpha)=A \\
& \frac{d}{d t}\left(T^{-1}(\alpha)\right) T(\alpha) \\
& \quad=k_{0} \operatorname{diag}\{m, m+1, m+2, \ldots, m+n-1\} .
\end{aligned}
$$

Substituting the above identities into (23) results in

$$
\begin{aligned}
\dot{y}= & A_{1}\left(1+\frac{\bar{k}_{0} e_{0}}{k_{0} \alpha}\right) y+\alpha^{-m} b u_{1} \\
& +T^{-1}\left(x_{0}\right)\left(-k_{0} \alpha\left(1+\frac{\bar{k}_{0} e_{0}}{k_{0} \alpha}\right) R_{2}+R_{1} u_{1}\right),
\end{aligned}
$$

where $A_{1}$ is defined in (13).

The second control input is designed as

$$
u_{1}=-\alpha^{m} K y=-\alpha^{m} K T^{-1}(\alpha) x,
$$

where $K=\left[k_{1}, k_{2}, \ldots, k_{n}\right]$ is a control gain row vector selected such that $A_{1}-b K$ is Hurwitz.

The closed-loop system of (25) and (26) becomes

$$
\begin{aligned}
\dot{y}= & \left(A_{1}-b K+\frac{\bar{k}_{0} e_{0}}{k_{0} \alpha} A_{1}\right) y \\
& -T^{-1}(\alpha)\left(k_{0} \alpha\left(1+\frac{\bar{k}_{0} e_{0}}{k_{0} \alpha}\right) R_{2}+R_{1} \alpha^{m} K y\right) \\
= & \left(A_{1}-b K+\frac{\bar{k}_{0} e_{0}}{k_{0} \alpha} A_{1}\right) y+R^{*},
\end{aligned}
$$

where

$$
R^{*}=-T^{-1}(\alpha)\left(k_{0} \alpha\left(1+\frac{\bar{k}_{0} e_{0}}{k_{0} \alpha}\right) R_{2}+R_{1} \alpha^{m} K y\right) .
$$

In view of (5), the converted residual term $R^{*}$ can be shown to be bounded by

$$
\begin{aligned}
\left\|R^{*}\right\|_{2} \leq & \left\|T^{-1}\right\|_{2} k_{0}|\alpha|\left|1+\frac{\bar{k}_{0} e_{0}}{k_{0} \alpha}\right|\left\|R_{2}\right\|_{2} \\
& +\left\|T^{-1}\right\|_{2}\|K\|_{2}|\alpha|^{m}\|y\|_{2}\left\|R_{1}\right\|_{2} \\
\leq & \left\|T^{-1}\right\|_{2} r_{2} k_{0}|\alpha|\left|1+\frac{\bar{k}_{0} e_{0}}{k_{0} \alpha}\right|\|x\|_{2}^{2} \\
& +\left\|T^{-1}\right\|{ }_{2} r_{1}\|K\|_{2}|\alpha|^{m}\|x\|_{2}\|y\|_{2} \\
\leq & r_{2} k_{0}\left|1+\frac{\bar{k}_{0} e_{0}}{k_{0} \alpha}\right||\alpha|\left\|T^{-1}\right\|_{2}\|T\|_{2}^{2}\|y\|_{2}^{2} \\
& +r_{1}\|K\|_{2}|\alpha|^{m}\left\|T^{-1}\right\|_{2}\|T\|_{2}\|y\|_{2}^{2} \\
= & h\left(\alpha, e_{0}\right)\|y\|_{2}^{2}
\end{aligned}
$$

with $h\left(\alpha, e_{0}\right)$ defined as

$$
\begin{aligned}
& h\left(\alpha, e_{0}\right) \\
& \cong r_{2} k_{0}\left|1+\frac{\bar{k}_{0} e_{0}}{k_{0} \alpha}\right| \max \left\{|\alpha|^{m+1},|\alpha|^{m-n+2}\right\} \\
& \quad \times \max \left\{1,|\alpha|^{2(n-1)}\right\} \\
& \quad+r_{1}\|K\|_{2} \max \left\{|\alpha|^{m},|\alpha|^{m-(n-1)}\right\} \max \left\{1,|\alpha|^{n-1}\right\} .
\end{aligned}
$$

As $\alpha, e_{0} / \alpha=\left(e_{0} / \alpha_{0}\right) e^{-\left(\bar{k}_{0}-k_{0}\right) t}$ are both bounded uniformly with $t$, and $h\left(x_{0}, e_{0}\right)$ is thus uniformly bounded provided $m-(n-1) \geq 0$. Since $A_{1}-b K$ is Hurwitz and $e_{0} / \alpha$ converges to zero exponentially, system $\dot{y}=\left(A_{1}-b K+\right.$ $\left.\left(\bar{k}_{0} e_{0} / k_{0} \alpha\right) A_{1}\right) y$ is globally exponential stable, and hence the perturbed system $\dot{y}=\left(A_{2}+\left(\bar{k}_{0} e_{0} / k_{0} \alpha\right) A_{1}\right) y+R^{*}$ is locally exponential stable by Lyapunov indirect approach [20].

Based on the above analysis, we arrive at the following results.

Proposition 2. Suppose that $\alpha=\alpha(t)=\alpha_{0} e^{-k_{0} t}, \alpha_{0} \neq 0, \bar{k}_{0}>$ $k_{0}>0, m \geq n-1, K$ is selected such that $A_{1}-b K$ is Hurwitz, then the following control law

$$
u_{0}=-k_{0} \alpha-\bar{k}_{0}\left(x_{0}-\alpha\right), \quad u_{1}=-\alpha^{m} K y
$$

guarantees that the states $x_{0}(t), u_{0}(t)$ globally converge to zero exponentially and $x(t), u_{1}(t)$ converge to zero exponentially for a sufficiently small $\|y(0)\|_{2}$.

Proof. It is obvious that $x_{0}(t), u_{0}(t)$ globally converge to zero exponentially. As $A_{1}-b K$ is Hurwitz and $\left\|R^{*}\right\|_{2} \leq$ $h\left(x_{0}, e_{0}\right)\|y\|_{2}^{2}$ with $h\left(x_{0}, e_{0}\right)$ uniformly bounded with $t$, the closed-loop system (27) is locally exponential stable, implying that $y(t), x(t)=T(\alpha(t)) y(t)$ and $u_{1}(t)=-\alpha^{m}(t) K y(t)$ are all convergent to zero exponentially for a sufficiently small $\|y(0)\|_{2}$.

Remark 5. Compared with the approach presented in [18] where the control law is continuous but not differentiable, 


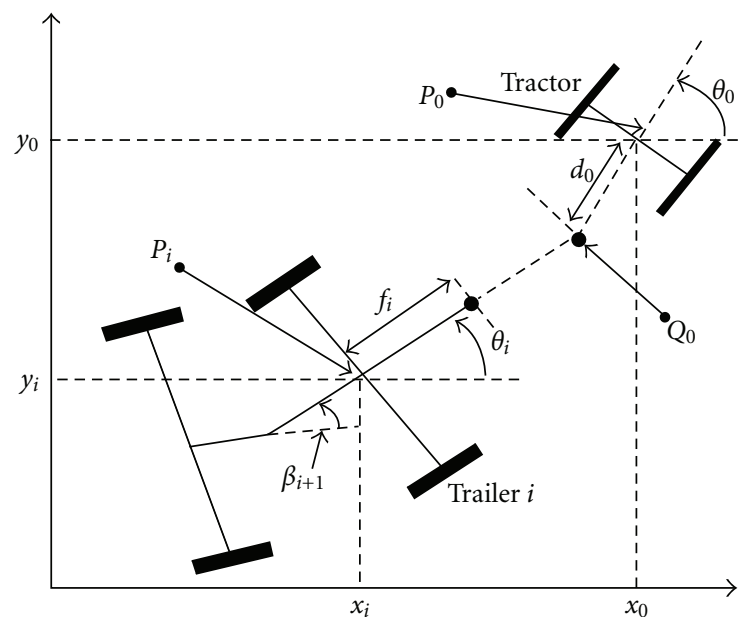

Figure 1: A tractor towing $n$ trailer with off-axle hitching.

the proposed control law (31) in this paper is smooth time varying and hence can be easily extended to include input dynamics of the approximate NCF (1)-(2) by one-step backsteeping.

\section{An Example: Local Exponential Regulation of an Off-Axle Tractor-Trailer}

Consider a tractor-trailer with a wheeled mobile tractor towing $n$ off-axle wheeled trailers shown in Figure 1, where $\left(x_{i}, y_{i}, \theta_{i}\right)$ denote the position and orientation of body $i$ ( $i=$ $0,1,2, \ldots, n),\left(v_{i}, \omega_{i}=\dot{\theta}_{i}\right)$ denote the linear and angular velocities of body $i(i=0,1,2, \ldots, n), \beta_{i}=\theta_{i-1}-\theta_{i}(i=$ $1,2,3, \ldots, n)$ represent the difference of orientation angles between body $i$ and body $i-1 . P_{i}(i=0,1,2, \ldots)$ is the center point on the wheel axle of body $i$ and $Q_{i-1}(i=1,2, \ldots, n)$ the connection point of body $i$ and body $i-1$. The distance between $P_{i}$ and $Q_{i}$ is $d_{i}$, and the distance between $P_{i}$ and $Q_{i-1}$ is $f_{i}$.

The kinematic equation of the tractor is

$$
\dot{x}_{0}=v_{0} \cos \theta_{0}, \quad \dot{y}_{0}=v_{0} \sin \theta_{0}, \quad \dot{\theta}_{0}=\omega_{0} .
$$

The kinematic relations of trailer $i$ can be derived as

$$
\begin{gathered}
v_{i}=v_{i-1} \cos \beta_{i}+d_{i-1} \dot{\theta}_{i-1} \sin \beta_{i}, \\
\dot{x}_{i}=v_{i} \cos \theta_{i}, \\
\dot{y}_{i}=v_{i} \sin \theta_{i}, \\
\dot{\theta}_{i}=\frac{1}{f_{i}}\left(v_{i-1} \sin \beta_{i}-d_{i-1} \dot{\theta}_{i-1} \cos \beta_{i}\right) .
\end{gathered}
$$

Select $x=\left[x_{0}, y_{0}, \theta_{0}, \beta_{1}, \beta_{2}, \ldots, \beta_{n}\right]^{T}$ as the state variables, and $u_{0}=v_{0} \cos \theta_{0}, \omega_{0}$ as the control inputs, the state equation can be derived from (32)-(33) as

$$
\begin{gathered}
\dot{x}_{0}=u_{0}, \\
\dot{x}=\left(A+R_{2}(x)\right) u_{0}+\left(b+R_{1}(x)\right) \omega_{0},
\end{gathered}
$$

where $R_{1}, R_{2}$ are high-order residual terms satisfying (5) and

$$
\begin{aligned}
& A=\left[\begin{array}{cccccc}
0 & 1 & 0 & 0 & \ldots & 0 \\
0 & 0 & 0 & 0 & \ldots & 0 \\
0 & 0 & -\frac{1}{f_{1}} & 0 & \ldots & 0 \\
0 & 0 & \frac{1}{f_{1}}\left(1+\frac{d_{1}}{f_{2}}\right) & -\frac{1}{f_{2}} & \ldots & 0 \\
\vdots & \vdots & \vdots & \vdots & \ddots & \vdots \\
0 & 0 & a_{1, n} & a_{2, n} & \ldots & -\frac{1}{f_{n}}
\end{array}\right],
\end{aligned}
$$

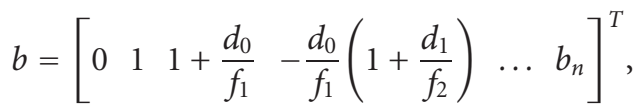

$$
\begin{aligned}
& a_{1, n}=(-1)^{n-2} \frac{d_{n-2} \times d_{n-3} \cdots \times d_{2} \times d_{1}}{f_{n-1} \times f_{n-2} \cdots f_{1}}\left(1+\frac{d_{n-1}}{f_{n}}\right) \text {, } \\
& a_{2, n}=(-1)^{n-3} \frac{d_{n-2} \times d_{n-3} \cdots \times d_{3} \times d_{2}}{f_{n-1} \times f_{n-2} \cdots f_{2}}\left(1+\frac{d_{n-1}}{f_{n}}\right) \text {, } \\
& b_{n}=(-1)^{n-1} \frac{d_{n-2} \times d_{n-3} \cdots \times d_{1} \times d_{0}}{f_{n-1} \times f_{n-2} \cdots f_{1}}\left(1+\frac{d_{n-1}}{f_{n}}\right) \text {, }
\end{aligned}
$$

The control object can be stated as design control law $u_{0}(\cdot), \omega_{0}(\cdot)$ such that the states $\left(x_{0}, y_{0}, \theta_{0}, \beta_{1}, \beta_{2}, \ldots, \beta_{n}\right)$ of the closed-loop system (34)-(35) converge to zero exponentially.

To apply Propositions 1 and 2 obtained in Section 3, it is required to verify the controllability of $\{A, b\}$.

Lemma 1. Suppose that $d_{i}>0(i=0,1, \ldots, n-1)$ and $f_{i}>$ $0(i=1,2, \ldots, n)$, then $\{A, b\}$ is a controllable pair.

Proof. The lemma can be proved by verifying $\mathrm{PBH}$ criterion of linear systems and is omitted here for brevity.

Remark 6. As $\{A, b\}$ is controllable, it can thus be further converted to the canonical controllable form (4) by a linear transformation so that the tractor-trailers system (34)-(35) can be expressed in approximate NCF (1)-(2).

To illustrate the effectiveness of the proposed control approaches, a tractor towing one trailer is taken as a simulation example. The state equation in this special case can be explicitly obtained as

$$
\begin{gathered}
\dot{x}_{0}=v_{0} \cos \theta_{0}, \\
\dot{y}_{0}=v_{0} \sin \theta_{0}, \\
\dot{\theta}_{0}=\omega_{0}, \\
\dot{\beta}_{1}=-c_{1} v_{0} \sin \beta_{1}+\left(1+c_{2} \cos \beta_{1}\right) \omega_{0},
\end{gathered}
$$

where $c_{1}=1 / f_{1}, c_{2}=d_{0} / f_{1}$. 


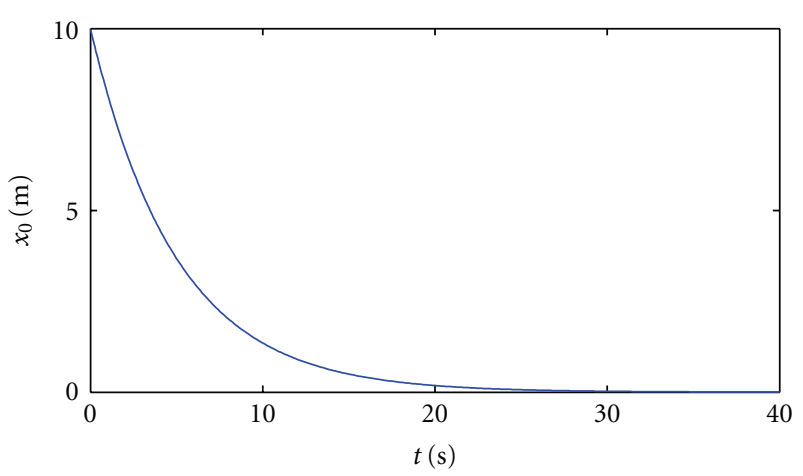

(a)

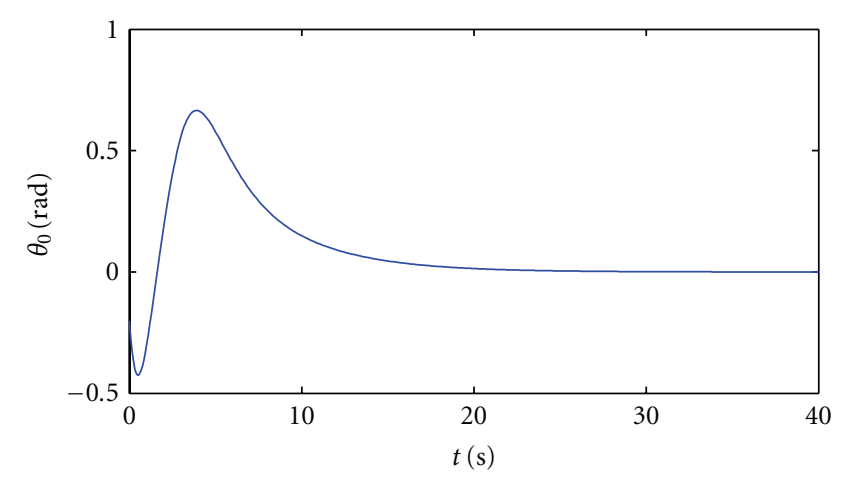

(c)

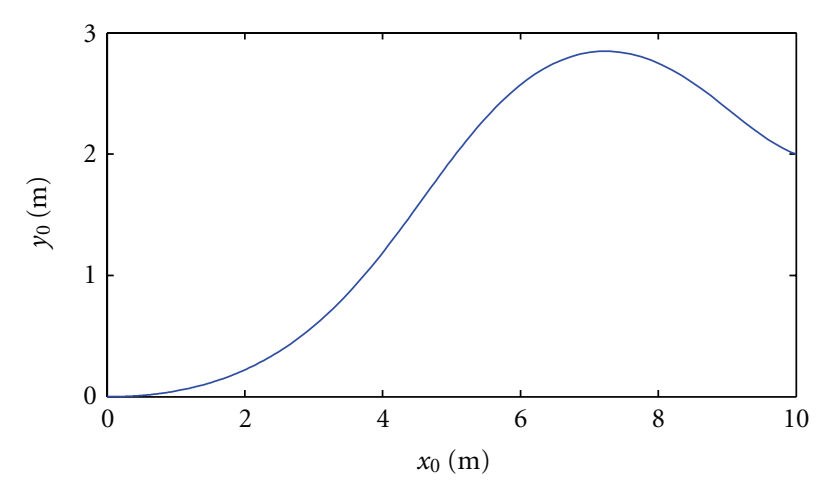

(e)

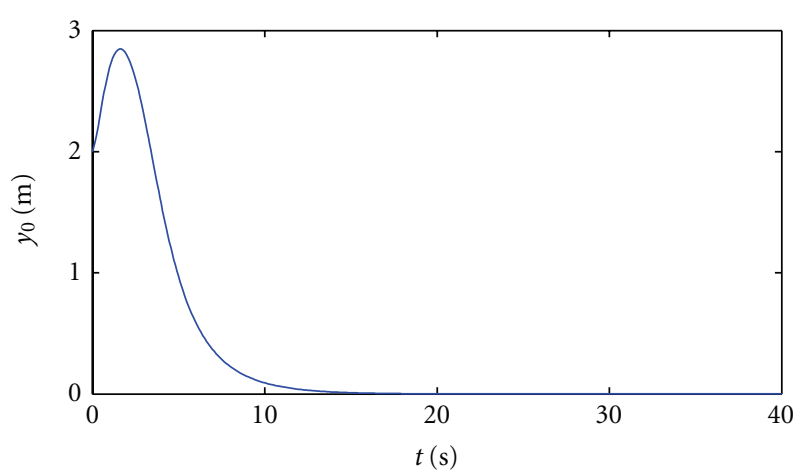

(b)

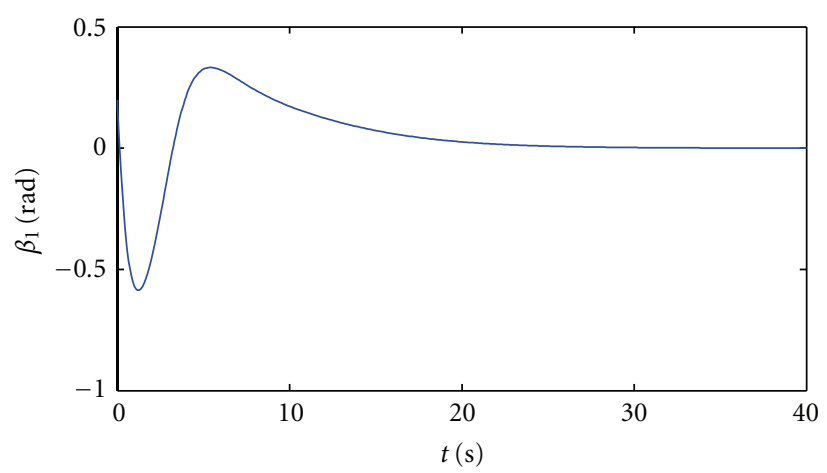

(d)

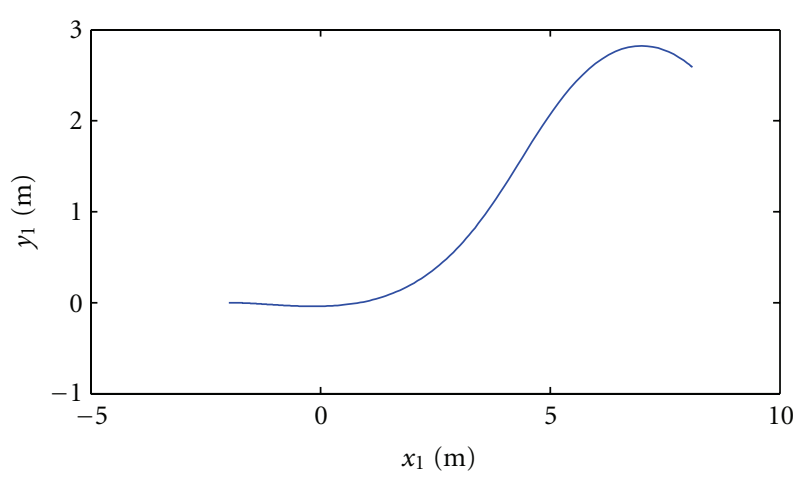

(f)

FIGURE 2: Time trajectories of states and geometric paths of the tractor and the trailer starting from the first initial state. tions:

Under the following coordinate and input transforma-

the state equation (38) is converted to the following form:

$$
\begin{aligned}
& x_{1}=c_{1}^{2} \beta_{1}, \\
& x_{2}=-c_{1} \beta_{1}+c_{1}\left(1+c_{2}\right) \theta_{0}, \\
& x_{3}=\beta_{1}-\left(1+c_{2}\right) \theta_{0}+c_{1}\left(1+c_{2}\right) y_{0}, \\
& u_{0}=v_{0} \cos \theta_{0}, \\
& u_{1}=c_{1}^{2}\left(-c_{1} v_{0} \sin \beta_{1}+\left(1+c_{2} \cos \beta_{1}\right) \omega_{0}\right) .
\end{aligned}
$$

$$
\begin{aligned}
& \dot{x}_{3}=\left(x_{2}+R_{23}\right) u_{0}+R_{13} u_{1}, \\
& \dot{x}_{2}=\left(x_{1}+R_{22}\right) u_{0}+R_{12} u_{1}, \\
& \dot{x}_{1}=u_{1}, \\
& \dot{x}_{0}=u_{0},
\end{aligned}
$$




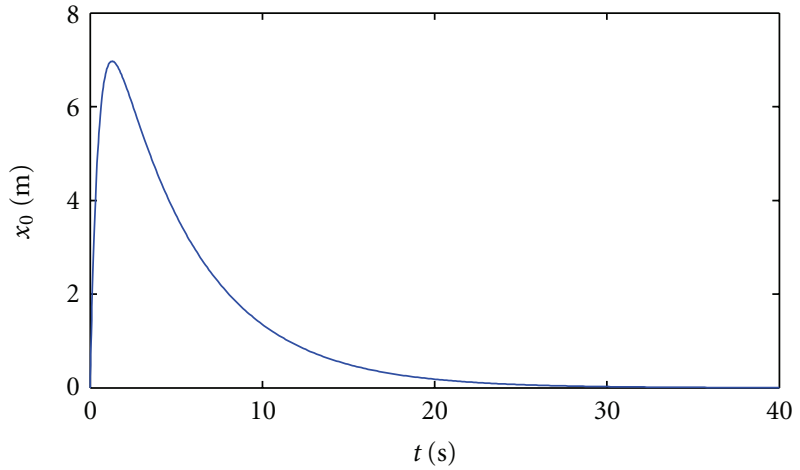

(a)

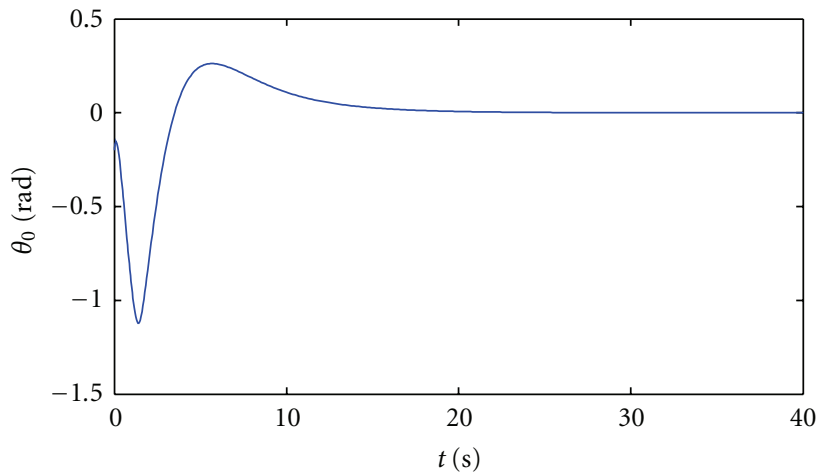

(c)

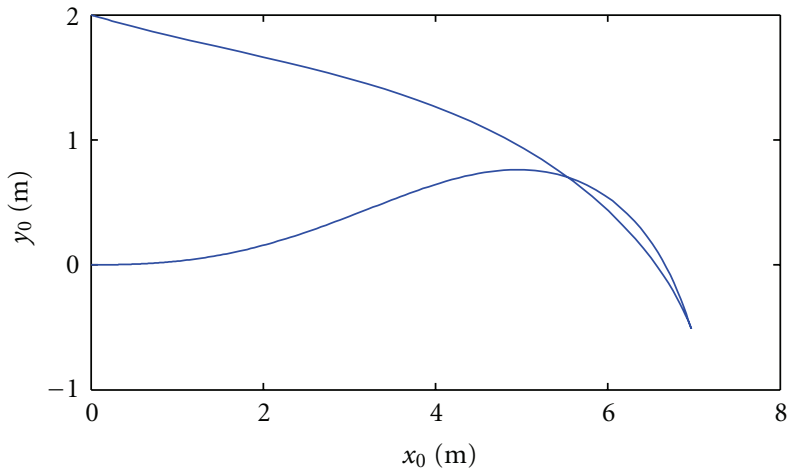

(e)

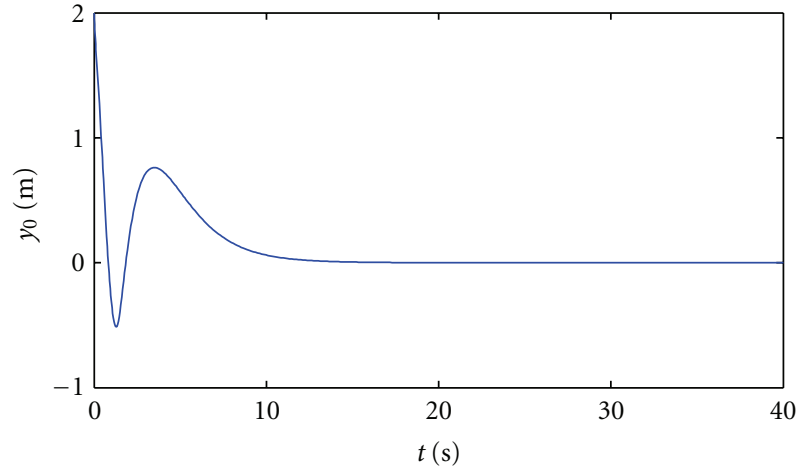

(b)

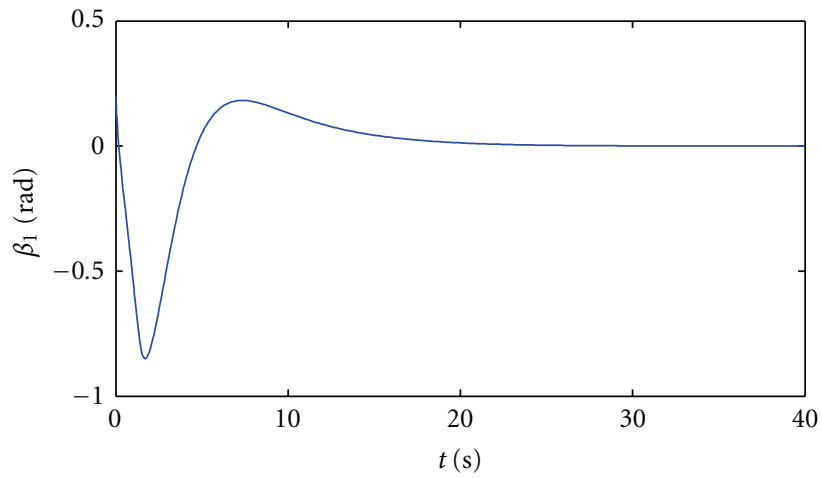

(d)

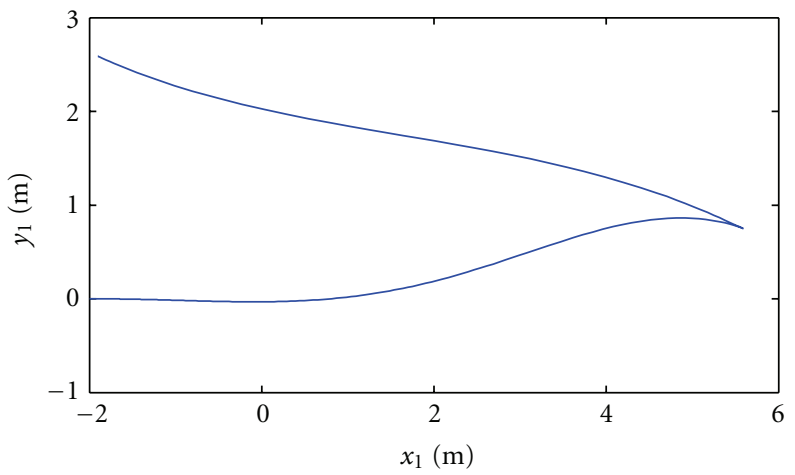

(f)

FIgURE 3: Time trajectories of states and geometric paths of the tractor and the trailer starting from the second initial state.

where

$$
\begin{aligned}
R_{23}= & c_{1}\left(-\left(\frac{\sin \beta_{1}}{\cos \theta_{0}}-\beta_{1}\right)+\left(1+c_{2}\right)\left(\frac{\sin \theta_{0}}{\cos \theta_{0}}-\theta_{0}\right)\right) \\
& +\frac{c_{1} c_{2}\left(\cos \beta_{1}-1\right) \sin \beta_{1}}{\left(1+c_{2} \cos \beta_{1}\right) / \cos \theta_{0}}, \\
R_{13}= & \frac{c_{2}\left(\cos \beta_{1}-1\right)}{c_{1}^{2} /\left(1+c_{2} \cos \beta_{1}\right)}, \\
R_{22}= & c_{1}^{2}\left(\frac{\sin \beta_{1}}{\cos \theta_{0}}-\beta_{1}\right)+\frac{c_{1}^{2} c_{2}\left(1-\cos \beta_{1}\right) \sin \beta_{1}}{\left(1+c_{2} \cos \beta_{1}\right) / \cos \theta_{0}}, \\
R_{12}= & \frac{c_{2}}{c_{1}\left(1-\cos \beta_{1}\right) /\left(1+c_{2} \cos \beta_{1}\right)} .
\end{aligned}
$$

In the state region $D=\left\{\left(x_{0}, y_{0}, \theta_{0}, \beta_{1}\right):\left|\theta_{0}\right| \leq \theta_{0 M}<\right.$ $\left.\pi / 2,\left|\beta_{1}\right| \leq \beta_{1 M}\right\},\left|R_{2 j}\right|(j=2,3)$ can be shown to be $O\left(\left\|\left(\theta_{0}, \beta_{1}\right)\right\|_{2}^{3}\right)$ and $R_{1 j}(j=2,3)$ to be $O\left(\left\|\left(\theta_{0}, \beta_{1}\right)\right\|_{2}^{2}\right)$.

The geometric parameters are set to $d_{0}=f_{1}=1$. The controller parameters are selected as $m=2, k_{0}=0.2, \alpha_{0}=$ $10, \bar{k}_{0}=2$, and $K=[1.92,-8.26,14.81]$ chosen such that the eigenvalues of $A_{1}-b K$ are assigned to $-(0.02,0.04,0.06)$.

The simulation is implemented for two initial states $\left(x_{0}(0), y_{0}(0), \theta_{0}(0), \beta_{1}(0)\right)=(10,2,-0.2,0.2)$ and $\left(x_{0}(0)\right.$, $\left.y_{0}(0), \theta_{0}(0), \beta_{1}(0)\right)=(0,2,-0.2,0.2)$. For the first initial state, where $x_{0}(0) \neq 0$, the control law (19) is applied; for the second initial state where $x_{0}(0)=0$, the control law (31) is applied. The time plots of state trajectories and geometric paths of the tractor and the trailer are shown in 
Figures 2 and 3 in respect to the two initial states. It is observed that the proposed control laws successfully regulate the state to zero from initial states and produce nice geometric paths for both the tractor and the trailer.

\section{Conclusion}

In this paper, we propose a discontinuous and a smooth time-varying control schemes for a class of nonlinear driftless systems in the approximated nonholonomic chained form, achieving local exponential convergence of state to the desired equilibrium point. The proposed control laws rely on the discontinuous and the smooth time-varying state transformations that convert the system to linear stable one perturbed by two- or higher-order terms of state. An application example of off-axle tractor-trailers is discussed in detail for illustrating the effectiveness of the proposed control approaches.

\section{Acknowledgments}

The paper is supported by National Science Foundation of China (no. 60874012). The author would like to thank the Editor and the reviewers for their helpful suggestions and careful review of the paper.

\section{References}

[1] I. Kolmanovsky and N. H. McClamroch, "Developments in nonholonomic control problems," IEEE Control Systems Magazine, vol. 15, no. 6, pp. 20-36, 1995.

[2] M. Fliess, J. Levine, P. Martin, and P. Rouchon, "Flatness and defect of non-linear systems: introductory theory and examples," International Journal of Control, vol. 61, no. 6, pp. 1327 1361, 1995.

[3] P. Martin and P. Rouchon, "Any (controllable) driftless system with 3 inputs and 5 states is flat," Systems and Control Letters, vol. 25, no. 3, pp. 167-173, 1995.

[4] R. W. Brockett, "Asymptotic stability and feedback stabilization," in Differential Geometric Control Theory, R. W. Brockett, R. S. Millman, and H. J. Sussmann, Eds., pp. 181-191, Birkhauser, Boston, Mass, USA, 1983.

[5] A. Astolfi, "Discontinuous control of nonholonomic systems," Systems and Control Letters, vol. 27, no. 1, pp. 37-45, 1996.

[6] Z. Sun and S. S. Ge, "Nonregular feedback linearization: a nonsmooth approach," IEEE Transactions on Automatic Control, vol. 48, no. 10, pp. 1772-1776, 2003.

[7] N. Marchand and M. Alamir, "Discontinuous exponential stabilization of chained form systems," Automatica, vol. 39, no. 2, pp. 343-348, 2003.

[8] C. Samson, "Control of chained systems application to path following and time-varying point-stabilization of mobile robots," IEEE Transactions on Automatic Control, vol. 40, no. 1, pp. 64-77, 1995.

[9] O. J. Sordalen and O. Egeland, "Exponential stabilization of nonholonomic chained systems," IEEE Transactions on Automatic Control, vol. 40, no. 1, pp. 35-49, 1995.

[10] P. Morin and C. Samson, "Control of nonlinear chained systems: from the Routh-Hurwitz stability criterion to timevarying exponential stabilizers," IEEE Transactions on Automatic Control, vol. 45, no. 1, pp. 141-146, 2000.
[11] C. Prieur and A. Astolfi, "Robust stabilization of chained systems via hybrid control," IEEE Transactions on Automatic Control, vol. 48, no. 10, pp. 1768-1772, 2003.

[12] I. Kolmanovsky, M. Reyhanoglu, and N. H. McClamroch, "Switched mode feedback control laws for nonholonomic systems in extended power form," Systems and Control Letters, vol. 27, no. 1, pp. 29-36, 1996.

[13] Z. P. Jiang, "Robust exponential regulation of nonholonomic systems with uncertainties," Automatica, vol. 36, no. 2, pp. 189-209, 2000.

[14] K. D. Do and J. Pan, "Adaptive global stabilization of nonholonomic systems with strong nonlinear drifts," Systems and Control Letters, vol. 46, no. 3, pp. 195-205, 2002.

[15] Z. Xi, G. Feng, Z. P. Jiang, and D. Cheng, "A switching algorithm for global exponential stabilization of uncertain chained systems," IEEE Transactions on Automatic Control, vol. 48, no. 10, pp. 1793-1798, 2003.

[16] T. Floquet, J. P. Barbot, and W. Perruquetti, "Higher-order sliding mode stabilization for a class of nonholonomic perturbed systems," Automatica, vol. 39, no. 6, pp. 1077-1083, 2003.

[17] E. Valtolina and A. Astolfi, "Local robust regulation of chained systems," Systems and Control Letters, vol. 49, no. 3, pp. 231238, 2003.

[18] D. A. Lizárraga, P. Morin, and C. Samson, "Chained form approximation of a driftless system. Application to the exponential stabilization of the general N-trailer system," International Journal of Control, vol. 74, no. 16, pp. 1612-1629, 2001.

[19] H. Date, M. Sampei, M. Ishikawa, and M. Koga, "Simultaneous control of position and orientation for ball-plate manipulation problem based on time-state control form," IEEE Transactions on Robotics and Automation, vol. 20, no. 3, pp. 465-479, 2004.

[20] H. K. Khalil, Nonlinear Systems, Prentice-Hall, Upper Saddle River, NJ, USA, 2nd edition, 1996. 

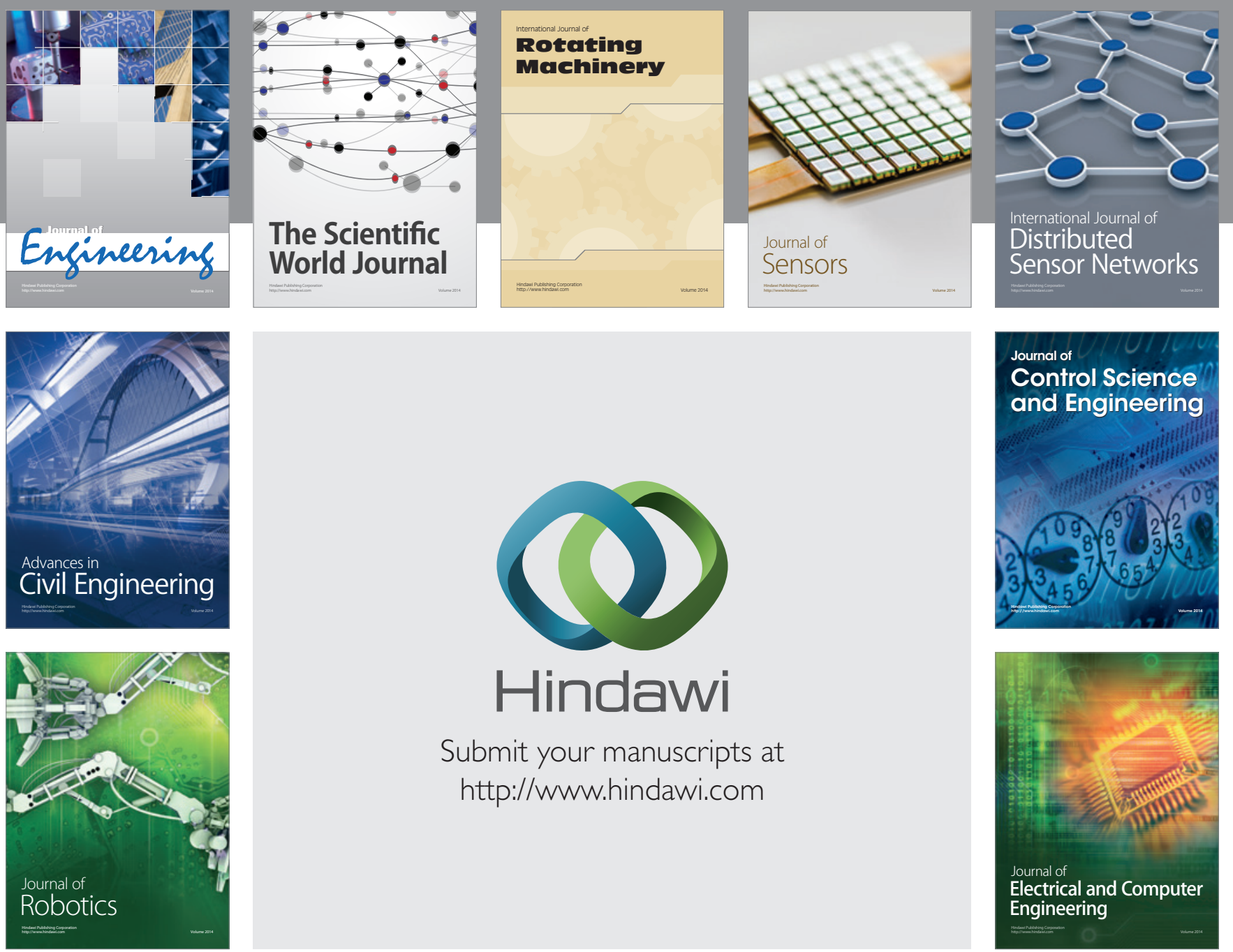

Submit your manuscripts at

http://www.hindawi.com
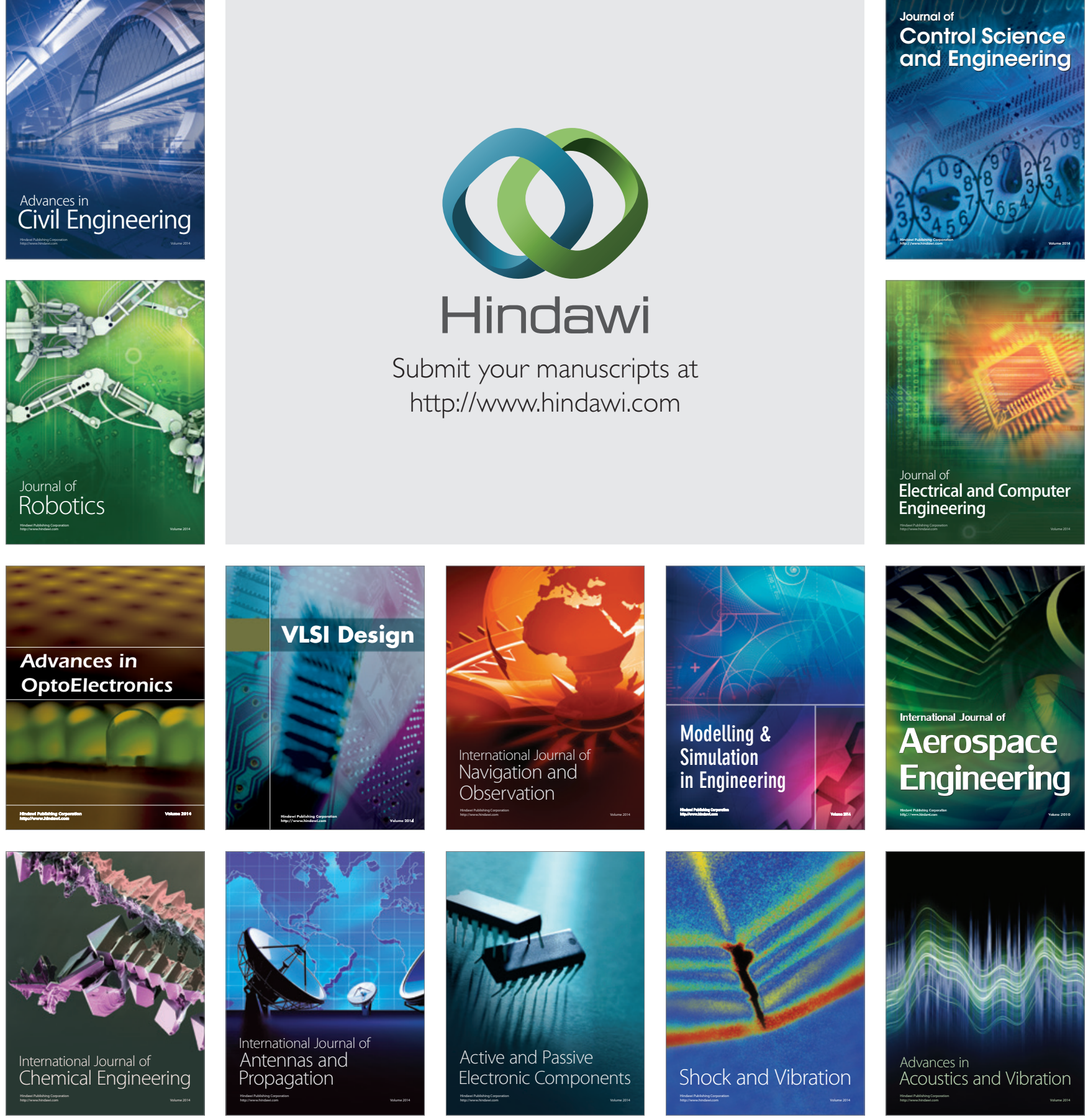